Meta

Journal des traducteurs

Translators' Journal

\title{
Les sous-titres... un mal nécessaire
}

\section{Lucien Marleau}

Volume 27, numéro 3, septembre 1982

URI : https://id.erudit.org/iderudit/003577ar

DOI : https://doi.org/10.7202/003577ar

Aller au sommaire du numéro

Éditeur(s)

Les Presses de l'Université de Montréal

ISSN

0026-0452 (imprimé)

1492-1421 (numérique)

Découvrir la revue

Citer cet article

Marleau, L. (1982). Les sous-titres... un mal nécessaire. Meta, 27(3), 271-285.

https://doi.org/10.7202/003577ar

Ce document est protégé par la loi sur le droit d'auteur. L'utilisation des services d'Érudit (y compris la reproduction) est assujettie à sa politique d'utilisation que vous pouvez consulter en ligne.

https://apropos.erudit.org/fr/usagers/politique-dutilisation/ 


\section{LES SOUS-TITRES... UN MAL NÉCESSAIRE*}

Lucien Marleau

Bien installé dans le creux d'un fauteuil chez moi... ou dans le fond de mon siège dans l'obscurité d'une salle de cinéma, les yeux rivés la plupart du temps au bas de l'écran, je regarde un film étranger; un film étranger présenté en version originale sous-titrée. Cela exige de moi un effort considérable pour suivre le film, pour admirer le jeu des interprètes, ou la beauté des images et, en même temps, essayer de percevoir dans son ensemble l'œuvre d'un grand cinéaste. Les difficultés sont nombreuses: - sous-titres parfois à moitié lus parce qu'ils sont projetés trop rapidement sur l'écran; - sous-titres parfois illisibles vu le même degré d'intensité lumineuse du sous-titre et de l'arrièreplan; - sous-titres qui partagent mon attention avec l'image puisqu'il faut bien en même temps suivre l'action...

Malgré le sombre tableau que je viens de brosser, force nous est d'admettre que, sans les sous-titres, il nous serait impossible de saisir l'essentiel d'un film, surtout si sa continuité filmique repose sur l'enchaînement verbal plutôt que sur le développement des images. Quoi qu'il en soit, sans s'interroger sur le bien-fondé des sous-titres, ou leur raison d'être, on les accepte la plupart du temps sans broncher - on les subit, car ils sont un mal nécessaire.

Pourquoi des sous-titres? Quelles en sont les origines? Qui les réalise? Comment les réalise-t-on? Nous allons tenter de répondre à ces questions. Pour ce faire, cependant, il est presque nécessaire de remonter aux origines mêmes du cinéma.

De 1890 à 1900, le cinéma intéresse surtout les inventeurs, les techniciens et les photographes: la mécanique de la caméra et du projecteur fait l'objet d'expériences. Il est normal d'ailleurs que, durant cette première décennie, l'on se préoccupe d'abord de l'aspect technique de la nouvelle invention.

Les films réalisés au cours de cette période ont un caractère documentaire. On enregistre fidèlement la réalité. Ainsi donc, la majorité des films tournés sont de pures reproductions de la réalité.

Peu à peu, les moyens techniques s'améliorent et, vers 1902, l'on s'attaque au contenu : on se rend compte que la caméra peut enregistrer autre chose que la réalité : on découvrait que l'on pouvait reproduire des actions, des histoires inventées de toutes pièces. Méliès fut l'un des premiers, en France, à modifier le sujet de ses films : il tourne de courtes saynètes, puis des histoires ou des petits drames burlesques. Vers le même temps, aux États-Unis, Edison et

* Colloque de Glendon, 1980. 
Porter tournent eux aussi des petits histoires fictives. En Angleterre, c'est Williamson, Smith, Paul et tous les autres, qui se livrent à des expériences de réalisation; cela aura comme résultat de modifier la façon de raconter.

Les films sont de plus en plus longs et compliqués. On doit alors recourir à des trucs pour justifier les changements de décor, de lieu et de temps. Ainsi naissent les intertitres. Dès 1903, Porter insère de brefs textes explicatifs entre les tableaux de son film - la Case de l'oncle Tom - pour faciliter la compréhension de l'action. Toutefois, la légende veut que ce soit Stuart Blackton qui, le premier, aux États-Unis, utilise les sous-titres pour résumer le dialogue. Dès le début du siècle, en ces temps héroïques du muet, l'absence du son était vivement ressentie comme une carence puisqu'on tentait déjà de la pallier par une musique d'ambiance. Les recherches effectuées en même temps en vue de l'enregistrement du son pour le cinéma n'aboutirent pas à un résultat valable. Le cinéma dut se contenter d'intertitres et de sous-titres jusqu'en 1927.

À l'époque, le cinéaste avait le choix entre deux méthodes de sous-titrage. Il pouvait interrompre une scène afin d'y insérer une ou plusieurs lignes de dialogue: c'était les sous-titres de dialogue, ou encore, il pouvait présenter une scène à l'aide d'une ou deux phrases explicatives: c'était les sous-titres de continuité ou explicatifs. Certains réalisateurs employaient les deux méthodes. Griffith préférait utiliser le sous-titre explicatif, car il n'aimait pas interrompre une scène par des dialogues. Il préférait surtout décrire ou interpréter l'action qu'il allait montrer, ou quelquefois, décrire l'action qu'il aurait dî̀ montrer. Quant à Ince, il optait pour les sous-titres de dialogue.

Mais les sous-titres indiquaient parfois autre chose, comme les changements de lieu - (...pendant ce temps, chez Ivan - meanwhile back at the ranch...) ou l'écoulement du temps, grâce à des adverbes, des locutions conjonctives, etc. (le lendemain... ou quelque temps plus tard...).

Selon le critique Marcel Martin, «...la plupart des films muets étaient bourrés d'intertitres dont le bavardage venait sans cesse interrompre la continuité des images».

\section{ÉVOLUTION ET DÉFINITION}

Le sous-titre n'a pas toujours été tel qu'on le connaît: au cours des années, il a subi des changements, il a évolué. On commença par de brèves descriptions ou explications insérées entre les plans ou les séquences. Puis, de courtes phrases de dialogue parlé dans le film furent ajoutées pour élucider certaines scènes.

Lorsque le cinéma devint parlant, un plus grave problème se posa: celui de l'internationalité du film. Bien des solutions furent alors envisagées pour l'aider à passer les frontières linguistiques: le sous-titrage et le doublage, ou postsynchronisation.

Le terme «sous-titre» fait son apparition dans le vocabulaire dès 1912. En effet, dans l'hebdomadaire parisien le Cinéma du 5 avril 1912, on lit: «Les 
sous-titres et les explications intercalés dans les films offrent quelquefois des surprises et des fautes d'orthographe et de sens».

Diamant-Berger, dans un article paru dans l'hebdo le Film, en date du 22 février 1919, parle de «subtitler», un américanisme, pour désigner le rédacteur de sous-titres d'un film à la place de «titreur» ou «titrier» qui commencent à être utilisés en 1921.

Quant aux termes «sous-titrage» et «sous-titrer», on les rencontre pour la première fois dans un article du 8 mars 1923 dans Mon ciné, un autre hebdo publié à Paris.

Aujourd'hui, lorsqu'il est question de ces différentes opérations, des mots plus précis, plus appropriés sont employés :

Le mot «intertitre » au temps du cinéma muet, désigne le texte explicatif inséré entre deux plans ou deux séquences pour faciliter la compréhension de l'action : ou pour résumer l'action ou le dialogue;

Le mot «doublage ou postsynchronisation» désigne l'opération consistant à substituer au texte dans la langue source un texte différent dans une autre langue. La postsynchronisation est donc l'opération généralement appliquée pour substituer au dialogue original la traduction dans la langue réceptrice.

Le mot «sous-titrage» désigne dans un film parlant étranger présenté en version originale, la traduction condensée du dialogue projetée au bas des images. Le sous-titrage consiste à traduire aussi fidèlement que possible un dialogue de film exprimé dans une langue plus ou moins ignorée du public. La traduction s'effectue au moyen d'une brève apparition à l'écran d'une inscription lumineuse rédigée dans la langue réceptrice.

Les intertitres sont quelquefois utilisés, même aujourd'hui. Un carton-titre est inséré entre deux séquences pour indiquer un changement de lieu ou un écoulement du temps. Ou encore, les mèmes mentions peuvent être placées en surimpression sur les premières images de la nouvelle séquence. Cette dernière méthode est préférable étant donné que, d'une part, elle n'interrompt pas la continuité visuelle et, d'autre part, le sous-titre est mieux intégré au film.

Mais, soit dit en passant, aujourd'hui les intertitres sont parfois employés pour donner au film une touche «antique», une saveur du temps du muet, mais souvent aussi pour créer des effets comiques ou parodier le cinéma muet, pour préciser un détail important qui ne peut être présenté autrement.

\section{Les formes de langage dans le film}

Je pense que l'on peut affirmer que, dans la version originale d'un film, on retrouve généralement le langage sous deux formes différentes :

1) Le langage sous sa forme sonore: il s'agit du dialogue original parlé ou dit dans le film et représenté sur la piste optique du film;

2) Le langage figuratif: c'est le système non verbal : cette forme de langage vient au secours du langage parlé. Il se manifeste lorsque le langage parlé n'est pas présent. C'est l'acteur qui exprime sa pensée à l'aide de gestes, d'attitudes ou de mimiques.

Il existe toutefois une troisième forme de langage qui prend en charge les éléments d'information véhiculés par l'image acoustique et les transforme en fait 
visuel, sinon le dialogue ne pourrait être perçu par le spectateur. Cette troisième forme de langage est :

3) Le langage sous sa forme visuelle: également système non verbal : c'est le dialogue original projeté de façon lumineuse au bas de l'écran.

Ces trois formes de langage contribuent à communiquer au spectateur la pensée des personnages par le truchement des acteurs.

\section{Fonctions linguistiques des sous-titres}

Loin d'être un caprice ou un luxe dans les versions originales des films étrangers, les sous-titres ont des fonctions bien précises à remplir, sans quoi ces films ne seraient pas perçus comme il se doit, étant donné l'absence d'autres paramètres pour comprendre le film.

Ces fonctions sont multiples:

1) Fonction de remplacement. En même temps que la traduction du dialogue s'opère d'une langue à une autre, le dialogue devient fait visuel en remplacement de l'image acoustique. En d'autres termes : le texte écrit remplace le texte parlé.

2) Fonction de communication. Les sous-titres transmettent de l'information destinée au récepteur dans le but de pallier un obstacle qui est la langue parlée dans le film. Il y a un émetteur, un message et un récepteur. C'est une communication médiatisée, indirecte et à sens unique.

3) Fonction émotive. Les sous-titres se proposent de susciter les mêmes émotions que celles suscitées par le dialogue dit dans le film. Le texte écrit véhicule l'affectivité de celui qui parle : ce qu'il manifeste ordinairement grâce au débit, à l'intonation et au rythme. Le fait que ce dialogue subisse, dans bien des cas, une compression, pourrait être un obstacle pour éveiller ces émotions.

4) Fonction d'ancrage. Les paroles précisent le sens de l'image, car celle-ci est polysémique la plupart du temps. L'ancrage du sens peut se faire au niveau d'un plàn ou de toute une séquence... comme le faisait Ince.

5) Fonction de relais. Ils prennent en charge des éléments d'information qui ne sont pas inclus dans l'image... comme le faisait Griffith.

6) Fonction de redondance. L'image et les sous-titres disent en gros la même chose. Si l'image, toutefois, dit les choses autrement, il y a redondance. Si le recouvrement est exact, il y a pléonasme.

\section{LES PROBLÈMES}

Au temps du muet, bien sûr, les films bourrés d'intertitres posaient des problèmes: le bavardage écrit, entre autres, venait sans cesse interrompre la continuité des images. Mais, en devenant sonores, les dialogues soulevaient aussi des problèmes: problèmes techniques, problèmes artistiques et esthétiques, problèmes physiologiques, problèmes psychologiques et problèmes linguistiques, problèmes tout aussi sérieux les uns que les autres. Quels sont ces problèmes? 


\section{Les problèmes techniques}

D'abord, les inscriptions surajoutées qui occupent la partie inférieure des images partagent le temps de perception entre le contenu de cette image et la lecture/compréhension du sous-titre. D'une part, le film original n'a pas été conçu et prévu pour un sous-titrage éventuel lors de sa réalisation et de son montage. D'autre part, un film qui aurait été prévu en fonction du sous-titrage, serait à priori une aberration du point de vue cinématographique, artistique, psychologique, etc.

Il ne restait qu'une solution : la fragmentation du dialogue en petites phrases courtes devant être lues et non entendues. Cette fragmentation même est, en soi, une aberration. Le traducteur de ces petites phrases courtes est limité par le temps, car la durée de projection des sous-titres est toujours fonction de la durée du dialogue dit. La traduction est également soumise aux contraintes d'un espace limité. Les dimensions de l'image sur la pellicule ne sont pas énormes, quoique le gigantisme de l'écran nous laisse illusoirement croire que l'espace réservé aux sous-titres est illimité.

Souvent l'emplacement d'un sous-titre correspond exactement à une plage claire (ou lumineuse) sur l'image, de même intensité lumineuse que le soustitre. Des lettres blanches sur un fond blanc ou gris pâle rendent la lecture impossible, même avec la meilleure volonté du monde. Le réalisateur n'a aucune raison de prévoir une plage sombre sur son image lors de la réalisation du film. La composition de l'image, l'éclairage, les éléments visuels, sont le résultat d'un parti-pris artistique de la part du réalisateur: ce parti-pris artistique, à son tour, est fonction des intentions et de la personnalité du cinéaste. Enfin, le cinéaste ne peut jamais prévoir ou anticiper le sort réservé à son film car c'est le public qui, en somme, consacre le film - c'est le public qui l'accepte ou le rejette parce qu'il aime ou n'aime pas le film.

Les problèmes que suscite l'utilisation d'un film sous-titré à la télévision sont encore plus cruciaux.

D'une part, problème d'espace dans l'image qui est grandement amputée dans sa périphérie par le tube cathodique : tout près de $20 \%$ de l'image sont perdus. Et, par le fait même, problème d'emplacement des sous-titres à l'intérieur de l'image. L'on se voit obligé d'empiéter davantage sur la partie de l'image visible à l'écran, empiètement de la partie vitale de l'image, si l'on ne veut pas perdre les débuts et les fins de phrases ainsi que la deuxième ligne des soustitres.

À cause de la réduction de toute la surface de l'image à la grandeur du tube du téléviseur, la grosseur du caractère des lettres pose également un problème de perception. Le caractère des lettres doit être plus gras afin d'être lisible au petit écran. Enfin, leur emplacement dans l'image est à considérer sérieusement.

Les conditions de visionnement de la télévision chez soi laissent souvent à désirer, réduisant davantage la lisibilité des sous-titres.

Les problèmes physiologiques

La compréhension du texte doit d'abord passer par l'œil. Pour ne pas accuser de retard dans le déroulement du film, la compréhension doit se faire 
presque instantanément avec la lecture du texte, sans perdre quoi que ce soit de l'action et tout en écoutant l'intonation de la voix du ou des personnages. La rapidité de succession des titres nous permet-elle toujours le temps de lire et de comprendre le texte? Ce temps de lecture n'est pas dicté par le degré d'acculturation du spectateur, ni basé sur son aptitude de lecteur rapide, en général. Or, nous savons que le temps de lecture visuelle d'un texte écrit est sensiblement plus long que celui de perception auditive du même texte exprimé de vive voix et débité à une cadence normale.

Pour permettre à notre œil de lire un sous-titre, il faut un certain nombre minimum d'images successives, impressionnées par un même texte. Ce minimum est déterminé par le temps nécessaire à l'œil humain pour lire un seul mot de 5 à 8 lettres. Ce temps est à peu près d'une seconde, temps que dure précisément le déroulement de 24 images, soit $1^{11 / 2}$ pied (ou $47 \mathrm{~cm}$ ) de $35 \mathrm{~mm}$ ou $71 / 4$ pouces (ou $18,3 \mathrm{~cm}$ ) de $16 \mathrm{~mm}$.

La longueur du texte en sous-titre est limitée par la durée rationnelle maximum de l'effort visuel et cérébral que l'on peut demander au spectateur pour lire un sous-titre, effort à fournir en plus de celui qui lui est imposé par le spectacle même. Cette durée maximale est de 6 secondes, temps de déroulement de 144 images.

Pour les personnes qui connaissent la langue parlée dans le film, les sous-titres sont non seulement superflus, mais déroutants : ils ont en effet le don de troubler leurs sens visuel et auditif en les empêchant de s'adonner librement à l'image et à la parole du film. Heureusement, ce n'est pas le cas de la majorité des spectateurs.

Les apparitions et les disparitions brusques et fréquentes des inscriptions lumineuses font subir à l'œil une longue série de chocs visuels, si l'on songe que le nombre moyen de sous-titres dans un long métrage est de 900 , dont chacun doit surgir et s'éclipser presque aussitôt: cela représente, à raison de deux par sous-titre, 1800 chocs par film. Ainsi, un film d'une durée de 2 heures, soit 7200 secondes, assène au spectateur un choc visuel environ toutes les 3 secondes.

\section{Les problèmes psychologiques}

L'effort demandé au spectateur est quand même considérable si l'on songe à l'impact psychologique des sous-titres: le partage du temps de perception, partage de la vigilance et de la participation au stage de l'émotivité, selon Gilbert Cohen-Séat. Comment l'opposition d'un donné sonore et d'un donné visuel discordants peut-elle enjoindre le spectateur moyen à l'empathie, à l'implication, au processus d'identification avec le protagoniste du film? N'est-ce pas trop exiger? N'est-ce pas un travail exténuant, un exercice au-delà de ses moyens que le spectateur n'est pas prêt à faire? Il a déjà payé son entrée; il cherche à s'évader de ses soucis journaliers, à passer une soirée agréable, à se faire distraire. Il ne demande rien d'autre.

Avec les apparitions et les disparitions brusques de quelque 900 soustitres, est-il possible de communiquer l'ambiance ou l'impact du film? Est-il possible d'apprécier un film à sa juste valeur? L'énorme effort exigé du 
spectateur moyen n'est pas de nature à encourager la détente, le confort d'une projection normale. Il en sort plus fatigué qu'avant.

Les impondérables du montage des diverses séquences parlées du film viennent également ajouter aux nombreux chocs visuels, dans les cas où spécifiquement les changements de plan interviennent en plein débit du dialogue. En effet, l'étalement d'un même sous-titre «à cheval» sur deux plans en coupure franche, produit sur l'œil la fầcheuse impression d'une apparition d'un nouveau sous-titre, alors qu'en réalité, c'est le même qui surgit à nouveau au moment du changement de plan. Le spectateur recommence à lire et perd un temps précieux, avant de s'apercevoir que le texte n'est pas changé.

Le spectateur d'un film sous-titré est frustré à priori d'une bonne partie du plaisir pour lequel il vient au cinéma.

En attendant un système sonore multilingue qui permettra à un public hétérogène d'entendre les dialogues d'un film chacun dans sa langue natale, rendonsnous à l'évidence: le sous-titrage est un mal nécessaire.

\section{Les problèmes artistiques et esthétiques}

Le grand art de transposer un dialogue parlé en sous-titrage visuel consiste à exprimer le maximum d'idées dans la compression avec le maximum de naturel dans l'artifice.

Pour que le temps de lecture visuelle et celui de la perception auditive du même texte soit égalisé et un équilibre assuré entre le dialogue et sa traduction, il convient de rendre la lecture de celle-ci moins longue. On ne peut l'obtenir que par voie de compression du texte initial qui se voit réduit à une plus simple expression.

Cela pose certains problèmes d'ordre esthétique, entre autres, le respect du caractère de l'œuvre originale. Il va sans dire que la compression du texte initial doit être effectuée sans le moindre préjudice pour la clarté de l'idée centrale soutenue dans le dialogue. Or, ce résultat n'est pas toujours facile à obtenir et l'on doit souvent recourir à de savants artifices pour y arriver. Mais le sort du film dépend souvent de la qualité du sous-titrage.

En réduisant le texte à une plus simple expression, on s'expose parfois à amputer le film de ses qualités inhérentes comme de son style, de sa personnalité, de sa clarté, de sa progression dramatique naturelle, de son rythme, de son équilibre dans la synthèse image/son. Il s'agit de sauvegarder au maximum la valeur scénique et photographique de l'image.

Un littérateur-adapteur médiocre pourrait faire une mauvaise adaptation d'un texte, tout comme un traducteur pourrait trahir l'ouvre originale par une traduction inexacte du texte, par une fragmentation arbitraire ou peu judicieuse du dialogue en une série de petites phrases, ou ne correspondant pas exactement au fragment du texte prononcé durant l'apparition du sous-titre, ou manquant de synchronisme avec le texte parlé ; ce sont des aberrations produites par des personnes plus ou moins qualifiées. Des problèmes techniques, peut-être, mais qui relèvent la plupart du temps de la décision ou d'un certain parti-pris artistique de l'adaptateur. 
La vocation réaliste de la parole est conditionnée par le fait qu'elle est un élément d'identification des personnages au même titre que le costume, la couleur de la peau ou le comportement général: il y a donc une adéquation nécessaire entre ce que dit un personnage et comment il le dit, et sa situation sociale et historique. Car la parole est sens mais aussi tonalité humaine, et c'est pour cette raison que le doublage est une monstruosité qui, selon certains auteurs, n'a pas l'ombre d'une justification... C'est aussi une question de conscience artistique et de respect de l'ocuvre d'art. En d'autres termes, une phrase de dialogue ne peut prendre véritablement son sens que par la manière dont elle est dite par l'acteur et par la manière dont elle est associée ou non à d'autres signes ressortissant du jeu des acteurs : mimique, gestuelle, composition ou typage, etc. Des éléments extérieurs à son propre processus de signification peuvent connoter le dialogue : le rythme du débit, l'utilisation de la musique, la manière de dire, ce qui est dit, la situation dans laquelle la phrase est dite, le rapport entre l'image et le dialogue, les contraintes ou les exigences posées par le tipage, etc.

\section{Les problèmes linguistiques}

On a dit, précédemment, que le sous-titrage était un «mal nécessaire». Ce constat présuppose que le sous-titrage est quand même accepté pour ce qu'il est; que l'on est prêt à fermer les yeux sur tous les problèmes que nous venons d'exposer. Si l'on n'acceptait pas ces contraintes et aberrations, cela voudrait dire qu'il ne nous serait pas loisible de voir des films étrangers, que nous serions privés du plaisir de voir des films extraordinaires.

Pour nous permettre, cependant, de visionner ces films étrangers, sommes-nous prêts à fermer les yeux sur les problèmes linguistiques? Car le sous-titrage en pose également. Quels sont ces problèmes que rencontre le traducteur-adaptateur?

Pour pouvoir traduire la totalité des dialogues d'un film, l'adaptateur doit fractionner ceux-ci en une série de petites phrases dont le temps de lecture n'excède pas 6 secondes. Il doit déterminer le début et la fin de chacune de ces phrases. Il doit préparer un «digeste» sommaire de ce qui se passe à l'écran. Il doit se garder de tomber dans le mot-à-mot. Il doit éviter de changer l'ordre des phrases de l'original. Il n'a pas besoin de traduire certaines phrases généralement très courtes et intelligibles à quiconque par la seule vertu de l'action. Il doit se rappeler que certaines répliques peuvent se passer de sous-titrage telles que:

- les expressions courantes à caractère international;

- les formules visuelles de salutation, de politesse, d'affirmation, de négation, d'étonnement, exclamation, répliques téléphoniques, etc.;

- les interpellations par nom propre;

- les interpellations par nom commun à sonorité familière;

- toutes sortes de bouts de phrase à sens incomplet qui ne sont pas immédiatement achevés et qui relèvent plutôt de la mimique que du dialogue proprement dit; 
- les explications que le spectateur connaît déjà;

- le dialogue d'ambiance.

L'adaptateur doit regrouper la plus grande quantité de texte dans un même sous-titre à la condition toutefois :

- que la longueur globale des textes groupés ne dépasse pas 9 pieds (6 secondes);

- que le contenu du dialogue réuni ne porte, de préférence, que sur une seule idée principale;

- que le personnage qui parle s'adresse tout le long de la phrase à la même personne ou au même groupe de personnes.

L'adaptateur peut réaliser une économie substantielle de sous-titres :

- en substituant un seul sous-titre plus long à trois courts;

- ou alors en éliminant un sous-titre sur deux, notamment dans le cas d'un dialogue échangé en forme de questions/réponses. On se contente de ne sous-titrer que la réponse, de sorte que la question posée y soit comprise;

- en plaçant, parfois, dans un seul sous-titre, deux courtes répliques très rapprochées, dites par des personnages différents;

- par un truquage d'asynchronisme, en permettant l'allongement, par le début ou par la fin, de la durée d'un dialogue trop court, mais dont la traduction est indispensable. Cela consiste à faire apparaître le sous-titre un rien en avance, ou le faire disparaître en retard, par rapport à la parole.

L'adaptateur doit se rappeler que la longueur des sous-titres et l'intervalle résultant de leur emplacement sont deux facteurs qui ont un effet décisif sur la qualité du sous-titrage.

Il sait qu'un bon sous-titreur n'est pas nécessairement un bon écrivain, ou qu'un très bon prosateur peut faire un piètre sous-titreur. Le sous-titreur doit souvent faire des concessions qu'un écrivain authentique refuserait de faire pour des motifs de conscience professionnelle.

Il doit adopter un style cinématographique qui soit accepté de tous les spectateurs. Il doit faire face à des contraintes peu ordinaires : ainsi, la somme des lettres, des signes de ponctuation et des espaces entre les mots du soustitre, doit égaler 50 , c'est-à-dire ne pas dépasser $50 \mathrm{ou}$ s'en rapprocher le plus possible. Supposons qu'un acteur prononce, à une vitesse normale, une phrase de 20 mots. On peut admettre que si le sous-titre n'en contient que 14 ou 15 , le spectateur s'apercevra à peine de la "compression", ou la considérera comme négligeable. Si, par contre, le sous-titre ne contient que 5 ou 6 mots et reste collé sur l'écran pendant toute la durée des 20 mots, le spectateur a le temps de lire deux fois ou trois fois et se sent frustré.

L'adaptateur sait qu'il n'est pas vrai qu'un sous-titre doit être le plus court possible. Un sous-titre est déjà fatalement court en raison du peu d'espace sans qu'il soit besoin de le raccourcir davantage. Un sous-titre doit être le plus long possible, aussi long que le permettent les données du repérage. 
Si l'adaptateur est amené à répartir une seule phrase de repérage sur deux sous-titres successifs, le temps qui s'écoule entre la fin du premier et le début du deuxième dépasse un certain maximum admis par la persistance visuelle: le spectateur risque alors de ne plus saisir le rapport entre les deux sous-titres en question. La pratique veut que cet intervalle ne dépasse pas $1 \frac{112}{2}$ pied, soit une seconde.

\section{Recommandations}

Si on avait à faire des recommandations à un novice, quelles seraient-elles?

1 - Transposer fidèlement les dialogues d'un film sans trahir leur esprit et sans rien dissimuler au public.

2 - La brièveté du temps de lecture exige une compréhension des textes la plus aisée et la plus rapide possible.

3 - L'indice réel ne doit jamais dépasser 70 comme maximum; s'il ne comporte qu'un seul mot, son indice ne doit pas être inférieur à 4-5. Un sous-titre de 2-3 lettres n'a que rarement une raison d'être.

4 - $\mathrm{Ne}$ pas surcharger les textes de signes de ponctuation dont l'absence n'enlève rien à la clarté : les points d'exclamation et de suspension, par exemple. Le rôle du sous-titre est de traduire le sens d'une phrase et non l'inflexion de la voix de la personne.

5 - Le signe à employer à la fin d'une phrase non terminée et au début de celle qui la complète, chacune formant un sous-titre à part, est le trait d'union, et non les points de suspension qui indiquent que la phrase reste «suspendue».

6 - La présence du point signifie clairement que la phrase est complète.

7 - En principe, les mots imprimés entièrement en majuscules ne seront utilisés que pour traduire les titres, génériques, inscriptions diverses, entêtes de journaux, etc. Éviter d'utiliser les majuscules dans les soustitres parlés.

8 - Mettre les accents sur les majuscules, car leur omission risque d'allonger le temps de lecture.

9 - Le caractère italique dans les sous-titres est surtout employé lorsque les personnages qui parlent sont absents du décor - et non absents du champ. L'italique est d'un heureux effet quand il illustre des propos à caractère abstrait, évocateur, imaginaire, se situant en quelque sorte en dehors de l'action du film, comme dans le rêve, voix de la conscience, citations, pensées ou répliques déjà entendues et rappelées pour en souligner la portée, voix entendues à la radio, à la télévision, au téléphone, propos écoutés aux portes, narrations ou commentaires en "voix off», les chansons, certains documents, lettres, affiches, annonces, pancartes.

10 - Le fractionnement d'une phrase sur deux ou plusieurs sous-titres consécutifs peut poser des problèmes linguistiques. Rappelons-nous que chaque sous-titre apparaît sur l'écran séparément: il importe donc de lui assurer une structure individuelle qui ne laisse place à aucune méprise quant à son sens. Un sous-titre peut ne pas être achevé dans son idée, mais le fragment figurant sur l'écran doit être complet, parfaitement clair et appeler une conclusion à laquelle on s'attend. 
11 - Être prudent lorsqu'il s'agit d'inversion de mots, de groupes de mots ou de phrases entières. L'ordre des mots influe sur le jeu de l'acteur, fait varier ses intonations et impose à sa voix des inflexions directionnelles, ascendantes ou descendantes. Un sous-titre qui s'en écarte trop risque de frapper «à contre-temps» par rapport aux accents du dialogue original et aux gestes qui l'accompagnent. Le synchronisme entre le dialogue parlé et sa traduction est alors compromis. Il suffit de donner aux sous-titres une construction qui suive de près celle du dialogue.

12 - Il faut donner au spectateur l'illusion de tout comprendre sans lire les sous-titres.

\section{LES INCIDENCES ÉCONOMIQUES}

À cause de la réglementation établie dans certains pays, le sous-titrage a certaines incidences économiques, spécialement dans l'industrie du cinéma vu qu'il procure à une foule de gens des emplois largement rémunérés.

Le sous-titrage d'un film demande la collaboration d'un nombre de spécialistes et de techniciens tels que le repéreur, le traducteur, le littérateur-soustitreur ou adaptateur, en plus du producteur, du graphiste qui préparera les cartons-titres, de l'opérateur de la caméra d'animation qui filmera ces cartonstitres, de l'opérateur de la tireuse optique qui placera les sous-titres en surimpression sur les plans du film, en plus de tous les techniciens en laboratoire : étalonneur, inspecteur, techniciens à la tireuse, techniciens à la développeuse, projectionniste, distributeur et propriétaires de salle et leurs employés.

Sans doute, les emplois reliés au sous-titrage sont le repéreur, le traducteur et le littérateur/adaptateur. En quoi consistent les fonctions de chacun?

\section{Le repéreur}

La principale fonction du repéreur est de repérer les dialogues du film: c'est l'ensemble des manipulations tendant à déterminer les débuts et les fins de phrases partielles constituant les dialogues du film.

Le repérage comporte les diverses tâches suivantes

1 - Transcrire fidèlement tous les dialogues qui se trouvent sur la piste sonore du film, si une telle transcription n'a pas été fournie par le producteur.

2 - Si une telle liste complète des dialogues originaux existe, elle devrait être vérifiée par lui, en comparant la liste avec les dialogues dans le film pour s'assurer de leur conformité.

3 - Marquer dans la copie standard du film en version originale, tous les débuts et fins de phrases partielles de dialogue.

4 - Déceler le degré d'importance de chacune de ces phrases partielles pour déterminer si les répliques sont de première importance, de moindre importance, ou pas importantes du tout.

5 - Condenser les dialogues afin de les rendre plus courts et signifiants; en éliminer parfois pour éviter une rapide succession de sous-titres courts et saccadés - tout cela sans nuire à la clarté et à la compréhension du film. 
6 - Si les phrases sont trop longues, les fragmenter en phrases partielles en tenant compte de la ponctuation appropriée et de la durée de l'intervalle entre les deux.

7 - Utiliser un système de numérotage des dialogues parlés avec les sous-titres correspondants.

8 - Déterminer la longueur de chaque sous-titre en terme de nombre d'images ou de cadrages.

Quelles sont les qualifications que l'on doit exiger de la part du repéreur?

- Avoir une grande culture cinématographique.

- Être un monteur de film qualifié, familier de tous les domaines techniques et artistiques du cinéma.

- Connaître une ou deux langues étrangères, sinon à fond, au moins à un degré suffisant pour comprendre un dialogue de difficulté moyenne.

- Être capable de prendre des décisions au niveau de l'importance des dialogues, doivent-ils être traduits?

\section{Le traducteur}

La tâche principale du traducteur consiste à traduire la totalité des dialogues d'un film dans la langue du pays, à transposer de l'une à l'autre les expressions idiomatiques, à partir de la liste complète que lui fournit le repéreur. Les qualifications que l'on peut exiger du traducteur de dialogues sont à peu près les mêmes que l'on exige de tout bon traducteur.

L'adaptateur

Les principales fonctions du littérateur/adaptateur sont les suivantes :

- Adapter les dialogues en sous-titres.

- Réécrire au besoin certaines phrases.

- Faire concorder les sous-titres avec le dialogue parlé, dans l'espace et le temps disponible.

- Modifier, au besoin, le repérage pour améliorer le synchronisme.

La qualification principale que l'on pourrait exiger de l'adaptateur est qu'il soit un écrivain, un auteur.

\section{CHEZ NOUS}

La situation que je viens de décrire ne prévaut pas partout. Au Québec, les rôles de repéreur, traducteur et adaptateur sont remplis par une seule et même personne. Ç'a été le cas lorsque $\mathrm{j}$ 'ai fait les sous-titres, entre autres, du film de Don Owen, The Ernie Game, réalisé en 1967. Ici, c'est la coutume de cumuler les fonctions, alors qu'en Europe chaque fonction est remplie par des personnes différentes.

Soulignons que très peu de films sont sous-titrés au Canada, sauf à l'Office National du film, de temps à autre. La plupart des films sont sous-titrés chez Titra, à New York ou en Hollande.

Pour être viable et rentable, le métier de sous-titreur doit se situer au sein d'une industrie cinématographique solide, bien établie et ayant une continuité garantie, et non sporadique comme la nôtre. 


\section{L'ASPECT JURIDIQUE}

Contrairement à ce que l'on pourrait penser, la présentation des films étrangers en version originale, dans certains pays, n'est pas laissée aux caprices du premier venu : elle est réglementée par des lois dans le but de contingenter l'entrée ou le nombre de films étrangers dans ces pays; de sauvegarder le bien culturel qu'est la langue nationale en freinant l'invasion culturelle par le cinéma; et, enfin, de protéger l'industrie du cinéma pour éviter que la production nationale soit dans un état de dépendance - comme c'est le cas au Canada depuis l'invention du cinéma.

Ainsi, en France, dans le projet de réglementation établi en 1957, suivi d'un décret en 1961, une des clauses stipule que «sauf dérogation exceptionnelle, la projection des films étrangers doit être effectuée en version originale soustitrée ». De plus, la version originale est un impératif de classement «art et essai » pour les films étrangers de qualité permettant aux cinémas d'art et d'essai de bénéficier d'une détaxation partielle.

Qu'en est-il, plus près de nous, concernant la projection des films étrangers en version originale et la protection du bien culturel?

Au Canada, il n'y a aucune loi ; il n'y a aucun quota imposé aux distributeurs qui sont, pour la plupart, des étrangers; c'est le free for all, car nous sommes à la merci des multinationales, sauf pour le tirage des copies d'exploitation qui doit se faire ici.

$\mathrm{Au}$ Québec, il y a eu des tentatives pour réglementer la projection de ces films. Il n'est pas normal, par exemple, que dans une province francophone comme le Québec, la plupart des films étrangers (qu'ils soient américains, anglais, italiens, russes ou allemands) soient présentés en première en version originale ou en version originale sous-titrés en anglais. C'est une anomalie que les cinéastes québécois tentent de corriger depuis le premier projet de loi-cadre du cinéma, soumis au ministre des Affaires culturelles, le 29 octobre 1971. Ce projet de loi, de nature incitative, prévoyait entre autres :

...pour les films étrangers de qualitê, une aide sous forme de remboursement de frais de copie et/ou de publicité et/ou de sous-titrage en français fait en vue d'une sortie en salles.

...pour les films étrangers de qualité convenant aux enfants, une aide sous forme de remboursement de frais de copie, publicité ou frais de versions à la condition que ces travaux soient effectués au Québec.

Ce projet de loi fut refusé par le ministre des Affaires culturelles.

Quatre ans plus tard, un autre projet de loi sur le cinéma était élaboré sans la collaboration des cinéastes québécois, et sanctionné le 19 juin 1975.

Ce projet de loi $\mathrm{n}^{\circ} 1$, dans la section III, 07, intitulé «Doublage et soustitrage ", paragraphe 39 , en page 16 , prévoit la clause suivante :

...les règlements peuvent prescrire que les films appartenant aux catégories qu'ils indiquent soient, si la version originale n'est pas en français, obligatoirement accompagnés d'une version doublée ou sous-titrée en français, à défaut de quoi ils ne pourront être présentés pour classification. Le doublage et le sous-titrage doivent être effectués entièrement au Québec, sous réserve des exceptions prévues par règlement ou des ententes que le Ministre conclut avec d'autres gouvernements. 
Les articles 49 et 50 , section I «Création et mandat de l'institut » chapitre IV, l'institut québécois du cinéma, en pages 18 et 19 , stipulent que :

...l'institut doit, dans l'exécution de son mandat (entre autres) :

Paragraphe (c) - Stimuler et encourager les industries qui concourent à la production cinématographique au Québec, notamment, en matière de doublage et de soustitrage... par les moyens suivants

En plus des prêts, des placements et des garanties aux prêteurs et aux investisseurs :

- primes à la qualité et aux succès;

- subventions, y compris des subventions de déficit;

- prix d'excellence;

- réinvestissement par les bénéficiaires d'aide financière des profits qu'ils tirent des sommes avancées par l'institut;

- dans les cas prévus en paragraphe (E) de l'article 49, prêts, bourses et subventions.

Excellente loi, mais depuis qu'elle a été sanctionnée, elle n'a été que partiellement appliquée et les versions originales continuent d'être projetées sans soustitres français dans la majorité des cas. Aucun des programmes d'aides financières n'a été effectivement accordé. Mais la loi est là. C'est un début.

\section{LES PERSPECTIVES D'AVENIR}

Le moins que 1'on puisse dire pour l'instant c'est que les sous-titres sont bien installés et pour longtemps. Malgré tout, le sous-titrage est un moyen efficace permettant aux cinéphiles de voir et de comprendre le film étranger, tout en appréciant le contenu de la bande sonore originale. La version soustitrée permet aux cinéphiles de parfaire leur culture cinématographique tout en se tenant en contact avec la production étrangère récente.

Mais les choses n'en resteront pas là, c'est sûr. Déjà, l'Office National du film du Canada produit des films $16 \mathrm{~mm}$ comportant une piste multilingue, c'est-à-dire que le film, pour l'instant, comporte deux pistes, l'une en français, l'autre en anglais, et qu'il y a possibilités d'y inclure 4 autres pistes, pour un total de 6. Un jour, il y aura des projecteurs permettant un choix de lecture dans la langue de son choix. Imaginons les possibilités du format $35 \mathrm{~mm}$ et du $70 \mathrm{~mm}$. Voilà pour l'avenir technique des sous-titres.

Qu'en est-il de l'avenir pour les spécialistes qui veulent se tailler une carrière dans le domaine du sous-titrage? Le sous-titrage est un art ingrat, disait un auteur, car le propre de l'auteur de sous-titres est avant tout de ne pouvoir donner libre cours à son talent d'écrivain. Mais c'est dans la restriction des moyens que se révèle le maître, dit un proverbe allemand.

L'avènement du cinéma parlant a créé de nombreux métiers nouveaux, entre autres, celui d'autcur-cinéaste et d'auteur-sous-titreur. Le sous-titrage peut être considéré comme un «art mineur» mais, tout mineur qu'il soit, on ne saurait le pratiquer sans y avoir été initié et sans avoir atteint à une sorte d'habileté qui relève davantage de la spéculation que de l'art.

Le sous-titrage n'est en somme qu'un truquage cinématographique comme tous les autres. Mais exécuté de main de maître, il doit rester... invisible. 
Nouvelle de dernière heure :

Le ministère de la Santé et du Bien-être Canada vient d'annoncer la mise en place d'un centre d'adaptation de documents audiovisuels qui se propose comme objectif le sous-titrage de documents audio-visuels afin de les adapter aux besoins des déficients auditifs. On prévoit dans le cadre de ce programme étudier les possibilités de retransmission des sous-titres par ondes hertziennes et, éventuellement mettre sur pied un système de diffusion.

Nous avons parlé des sous-titres. Il y a également une autre question encore plus délicate, celle du doublage, qui n'est pas le sujet de nos propos ici. Si le sous-titrage pose des problèmes sérieux, attendez de voir ceux du doublage.

\section{BIBLIOGRAPHIE}

BATICLE, Yveline (1968): Manuel d'initiation au cinéma, Éditions Magnard, Paris.

COHEN-SÉAT, Gilbert (1958) : Essai sur les principes d'une philosophie au cinéma, Presse Universitaire de France, Paris.

DABORN, John (1960) : Cine Titling, Fountain Press, London.

DUCA, Lo (1963) : Technique du cinéma, Presses universitaires de France, Paris.

FORD, Charles et C. FONTUGNE, (1957): Tout sur le cinéma et la télévision, «Je sais» Mame, Paris.

LAKS, Simon (1957) : Le sous-titrage de films, Propriété de l'auteur, Paris.

LEBEL, J. Patrick (1971) : Cinéma et idéologie, Nouvelle Critique, Éditions Sociales, Paris. MARTIN, Marcel (1955) : le Langage cinématographique, "7 Art», Éditions du Cerf, Paris. MACGOWAN, Kenneth (1965) : Behind the Screen, A. Delacorte Press Book, New York.

ROGER, Jos : Naissance d'un film, Éditions Universitaires, Paris.

TABĖS, René (1969) : "L' "Affaire» de la version originale», dans Positif, $\mathrm{n}^{\circ}$ 104, avril, Paris, p. 45 .

BESSY, Maurice et Jean-Louis CHARDANS (1971): Dictionnaire du cinéma et de la télévision, tome IV, Jean-Jacques Pauvert éditeur, Paris.

Encyclopédie Universelle Illustrée.(1968): Les Éditions Maisonneuve, Montréal.

Projet de loi-cadre du cinéma soumis au ministre des Affaires culturelles, le 29 octobre 1971.

Gouvernement du Québec, Projet de loi $n^{\circ} 1$, sanctionné le 19 juin 1975. 